\title{
Erratum
}

\section{Renormalization Group Approach to the Surface and Defect Critical Behavior in the Potts Model}

\author{
P.M. Lam \\ Institute of Theoretical Physics, Chinese Academy of Sciences, Beijing, China \\ Z.Q. Zhang \\ Institute of Physics, Chinese Academy of Sciences, Beijing, China
}

After our paper 'Renormalization Group Approach to the Surface and Defect Critical Behavior in the Potts Model' has been published in Z. Phys. B - Condensed Matter 52, 315-320 (1983), we received a letter from Dr. C. Tsallis pointing out two misprints in our Eq. (6). It should read as follows

$g\left(t_{0}, t_{1}\right)=\frac{t_{1}^{2}+t_{0}^{2}+2 t_{1} t_{0}^{2}+(q-2)(q-3) t_{1}^{2} t_{0}^{3}+(q-2)\left(3 t_{1}^{2} t_{0}^{2}+2 t_{1} t_{0}^{3}\right)}{1+(q-1)\left(2 t_{1} t_{0}^{2}+t_{1}^{2} t_{0}^{2}\right)+(q-1)(q-2) t_{1}^{2} t_{0}^{3}}$

P.M. Lam

Institute of Theoretical Physics

Chinese Academy of Sciences

Beijing

China
Z.Q. Zhang

Institute of Physics

Chinese Academy of Sciences

Beijing

China 